\title{
Semigroups whose idempotents form a subsemigroup*
}

\author{
J. Almeida ${ }^{\dagger}$ J.-E. Pin and P. Weil ${ }^{\ddagger}$
}

August 17, 2005

\section{Sumário}

Prova-se que todo o semigrupo $S$ cujos idempotentes formam um subsemigrupo admite uma cobertura E-unitária com a mesma propriedade. Além disso, se $S$ é E-denso ou regular, então a sua cobertura pode ser escolhida como sendo do mesmo tipo. Enfim, descreve-se a estrutura dos semigrupos finitos E-unitários densos. Estes resultados estendem os de Fountain sobre semigrupos cujos idempotentes comutam, e os de Birget, Margolis e Rhodes, e Jones e Szendrei sobre E-semigrupos finitos.

\section{Résumé}

Nous montrons que tout semigroupe $S$ dont les idempotents forment un soussemigroupe admet un revêtement E-unitaire avec la même propriété. De plus, si $S$ est E-dense ou orthodoxe, alors son revêtement peut être choisi de même. Enfin, nous décrivons la structure des semigroupes E-unitaires denses. Nos résultats généralisent ceux de Fountain sur les semigroupes dont les idempotents commutent, et sont analogues à ceux de Birget, Margolis et Rhodes, et Jones et Szendrei sur les E-semigroupes finis.

We prove that every semigroup $S$ in which the idempotents form a subsemigroup has an E-unitary cover with the same property. Furthermore, if $S$ is E-dense or orthodox, then its cover can be chosen with the same property. Then we describe the structure of E-unitary dense semigroups. Our results generalize Fountain's results on semigroups in which the idempotents commute, and are analogous to those of Birget, Margolis and Rhodes, and of Jones and Szendrei on finite E-semigroups.

\footnotetext{
*The authors gratefully acknowledge support from the Working Group ASMICS of the European Community program ESPRIT (EBRA 3166).

†Centro de Matemática, Universidade do Porto, 4000 Porto, Portugal.

‡LITP, Université Paris 6 et CNRS, Tour 55-65, 4 place Jussieu, 75252 Paris Cedex 05, France.
} 


\section{Introduction}

This paper is part of a continuing investigation of the structure theory of semigroups whose first cornerstones were two theorems by McAlister on the theory of inverse semigroups $[11,12]$. The first of these theorems states that each inverse semigroup is covered by a regular extension of a group by a semilattice, where the covering map is one-to-one on idempotents. These regular extensions were later proved to be exactly the E-unitary inverse semigroups [16]. The second theorem was a representation theorem of the E-unitary inverse semigroups. McAlister $[11,12]$ gave a first description of these semigroups in terms of $P$ semigroups. Another representation for these semigroups is due to O'Carroll [15] who proved that they are exactly the regular subsemigroups of the semidirect products of a group by a semilattice.

These seminal results were generalized in several directions. In particular, Margolis and Pin [9] showed that a natural non-regular analogue of the class of inverse semigroups is the class of semigroups in which the idempotents commute. They proved a representation theorem for the E-unitary semigroups in this class, which extends McAlister's theorem. However this theorem was stated in a rather different way than the $P$-semigroup theorem. In the proof, Margolis and Pin made use of categories, considered as algebraic objects generalizing semigroups. In the same paper [9], they also proposed a conjecture to generalize McAlister's covering theorem to the non-regular case. This conjecture was first solved by Ash [1] for the finite case, and more recently by Fountain [5] for the general case, using totally different techniques.

Generalizing further, a natural generalization of semigroups in which idempotents commute is given by E-semigroups, that is, semigroups in which the idempotents form a subsemigroup. Regular E-semigroups, also known as orthodox semigroups, were widely studied, in particular by Szendrei [22, 24, 25], Kad̆ourek and Szendrei [7], Takizawa [27], and finite E-semigroups were studied by Birget, Margolis and Rhodes [3] and Jones and Szendrei [6].

In this paper we complete this study by proving two results on arbitrary E-semigroups. The first one states that every E-semigroup $S$ has an E-unitary cover, and that if $S$ is E-dense or regular, then its E-unitary cover can be chosen with the same property. The second one is a representation theorem for E-unitary E-semigroups. Our proof for the first theorem is essentially similar to Fountain's [5], even though the presentation is different. The proof of our second theorem is inspired by the proof of the analogous result of Margolis and Pin [9] and makes essential use of the concept of category. Note that in order to complete the picture it would be interesting to generalize 0'Carroll's representation theorem mentioned above to the case of E-unitary orthodox semigroups, and to extend Szendrei's [23, 24] results in this direction.

The paper breaks up into four main sections. The first one deals with basic properties of E-dense and E-unitary E-semigroups. The covering theorem is stated and proved in Section 2, while the representation theorem is proved in the third section. The last section, included here for the sake of completeness, contains a survey of the analogous results on finite E-semigroups. 


\section{Preliminaries}

Let $S$ be a semigroup. Then $S^{1}$ denotes the monoid constructed as follows: $S^{1}=S$ if $S$ is a monoid, and, if $S$ is not a monoid, $S^{1}=S \cup\{1\}$ where 1 is an identity.

If $S$ and $T$ are semigroups, a relational morphism $\tau: S \rightarrow T$ from $S$ into $T$ is a mapping from $S$ into the power set $\mathcal{P}(T)$ such that $s \tau \neq \emptyset$ and $(s \tau)\left(s^{\prime} \tau\right) \subset\left(s s^{\prime}\right) \tau$ for all $s, s^{\prime} \in S$. If $\tau$ is a relational morphism from $S$ into $T$, then the graph of $\tau$, that is, the set $R_{\tau}=\{(s, t) \in S \times T \mid t \in s \tau\}$, is a subsemigroup of $S \times T$ whose projection into its first component is onto $S$ (this constitutes an alternate definition of a relational morphism). Morphisms and inverses of onto morphisms are examples of relational morphisms. Relational morphisms are closed under composition. Let $\tau: S \rightarrow T$ be a relational morphism, and let $\alpha$ and $\beta$ be the projections from the graph $R_{\tau}$ of $\tau$ into $S$ and $T$ respectively. It is easy to verify that $\tau=\alpha^{-1} \beta$. This factorization is called the canonical factorization of $\tau$ (see $[19,18])$.

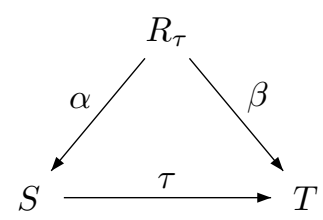

Let $X$ and $Y$ be subsets of $S$. We let

$$
\begin{aligned}
& X^{-1} Y=\{s \in S \mid \exists x \in X: x s \in Y\} \\
& X Y^{-1}=\{s \in S \mid \exists y \in Y: s y \in Y\} .
\end{aligned}
$$

A subsemigroup $T$ of $S$ is called unitary [2, 8] if $T^{-1} T \cap T T^{-1} \subset T$, that is, if, for all $t, t^{\prime} \in T$ and $s \in S, t s \in T$ and $s t^{\prime} \in T$ implies $s \in T$. The subsemigroup $T$ is called dense if every element $s$ of $S$ can be completed on the right and on the left into an element of $T$, that is, if there exist $s^{\prime}$ and $s^{\prime \prime}$ in $S$ such that $s s^{\prime} \in T$ and $s^{\prime \prime} s \in T$.

As usual, $E(S)$ denotes the set of idempotents of $S$. An E-semigroup (resp. an E-commutative semigroup) is a semigroup such that $E(S)$ is a subsemigroup (resp. a commutative subsemigroup) $)^{1}$.

An E-unitary semigroup (resp. E-dense semigroup, E-unitary dense semigroup) is a semigroup $S$ such that $E(S)$ is a unitary subsemigroup (resp. a dense subsemigroup, an unitary and dense subsemigroup). An orthodox semigroup is a regular E-semigroup. The next propositions summarize some elementary properties of E-semigroups and have been rediscovered many times. More properties of E-dense semigroups can be found for instance in Mitsch [14].

Proposition 1.1 Let $S$ be an E-semigroup and let $E=E(S)$. The following conditions are equivalent.

(1) $S$ is E-dense.

(2) For every $s \in S$, there exists $s^{\prime} \in S$ such that $s s^{\prime} \in E$.

(3) For every $s \in S$, there exists $s^{\prime \prime} \in S$ such that $s^{\prime \prime} s \in E$.

\footnotetext{
${ }^{1}$ This terminology is different from the one used in $[9,5]$, but seems to be more appropriate in this more general context.
} 
(4) For every $s \in S$, there exists $s^{\prime} \in S$ such that $s s^{\prime} \in E$ and $s^{\prime} s \in E$.

Proof. Clearly, (4) implies (1) and (1) implies (2) and (3). By symmetry, it suffices to show that (2) implies (4). Let $s \in S$ and let $s^{\prime} \in S$ be such that $s s^{\prime} \in E$. Then $s^{\prime} s s^{\prime} s \in E$ since $s^{\prime} s s^{\prime} s s^{\prime} s s^{\prime} s=s^{\prime}\left(s s^{\prime}\right)\left(s s^{\prime}\right)\left(s s^{\prime}\right) s=s^{\prime}\left(s s^{\prime}\right) s$. It follows that $\left(s^{\prime} s s^{\prime}\right) s \in E$ and $s\left(s^{\prime} s s^{\prime}\right) \in E$, proving (4).

Proposition 1.2 Let $S$ be an E-unitary semigroup, and let $E=E(S)$.

(1) For every $s, t \in S$, if st $\in E$, then $t s \in E$.

(2) For every $s, t \in S$ and $e \in E$, if $s t \in E$, then set $\in E$.

(3) For every $s, t \in S$ and $e \in E$, if set $\in E$, then $s t \in E$.

Proof. (1) Since $s t \in E$, tsts and $t$ ststs are idempotents. It follows that $t s$ lies in $E^{-1} E \cap E E^{-1}$, whence $t s \in E$, since $S$ is E-unitary.

(2) If $s t \in E$, then $t s \in E$ by (1), and ets $\in E$ since $S$ is an E-semigroup. Now ets $=(e t) s \in E$, and, by (1), set $\in E$.

(3) If set $\in E$, then $t(s e) \in E$ and (et) $s \in E$ by (1). It follows that $t s$ lies in $E^{-1} E \cap E E^{-1}$, whence $t s \in E$. Finally, st $\in E$ by (1).

Proposition 1.3 Let $S$ be an E-semigroup and let $E=E(S)$. The following are equivalent:

(1) $S$ is E-unitary.

(2) For every e, $s \in S$, the conditions e, es $\in E$ imply $s \in E$.

(3) For every $e, s \in S$, the conditions e, se $\in E$ imply $s \in E$.

Proof. Clearly, (2) or (3) implies (1). By symmetry, it suffices to show that (1) implies (2). Suppose that $S$ is E-unitary, and let $e, e s \in E$. Then, by Proposition 1.2, se $\in E$. It follows that $s \in E^{-1} E \cap E E^{-1}$ and $s \in E$ since $E$ is unitary.

Proposition 1.4 Let $S$ be an E-unitary dense semigroup and let $E=E(S)$. Then, for all $r, t \in S^{1}$ and $s \in S$, rst $=r t$ implies $s \in E$.

Proof. Suppose $r s t=r t$. Since $E$ is dense, there exists $r^{\prime}, t^{\prime} \in S$ such that $r^{\prime} r, t t^{\prime} \in E$. It follows that $\left(r^{\prime} r\right) s\left(t t^{\prime}\right)=\left(r^{\prime} r\right)\left(t t^{\prime}\right) \in E$. Since $S$ is E-unitary, it follows that $\left(r^{\prime} r\right) s \in E$ by Proposition $1.3(3)$ and $s \in E$ by Proposition 1.3 (2). by

Given a semigroup $S$, we denote by $\leq_{\mathcal{R}}$ and $\leq_{\mathcal{L}}$ the quasi-orders on $S$ defined

$s \leq_{\mathcal{R}} t \quad$ if and only if there exists $u \in S^{1}$ such that $s=t u$,

$s \leq_{\mathcal{L}} t$ if and only if there exists $u \in S^{1}$ such that $s=u t$.

Note that, if $e$ is an idempotent and $s \in S$, then $s \leq_{\mathcal{R}} e$ if and only if $e s=s$ and $s \leq_{\mathcal{L}} e$ if and only if $s e=s$. 


\section{The covering theorem}

Let $S$ and $T$ be semigroups. A surjective morphism $\varphi: T \rightarrow S$ which is oneto-one on idempotents (or "idempotent separating") is called a covering. In this case, $T$ is said to be a cover of $S$. The aim of this section is to prove the following theorem.

\section{Theorem 2.1}

(1) Every E-semigroup has an E-unitary cover.

(2) Every E-dense semigroup has an E-unitary dense cover.

(3) Every orthodox semigroup has an E-unitary orthodox cover.

Our proof is basically the same as the proof given by Fountain [5] in the case of E-commutative dense semigroups, but our presentation is slightly different. We first give a sufficient condition to ensure that an E-semigroup has an Eunitary cover, extending a result of [13] on inverse semigroups. Then we show that every E-semigroup satisfies this condition.

Proposition 2.2 Let $S$ be an E-semigroup. Suppose there exists a group $G$ and a relational morphism $\tau: S \rightarrow G$ such that $1 \tau^{-1}=E(S)$. Then $S$ has an E-unitary cover. Furthermore, if $S$ is E-dense and if $\tau$ satisfies the following condition

(*) for all $s \in S$ and $g \in s \tau$, there exists $s^{\prime}$ such that $s s^{\prime} \in E(S)$ and $g^{-1} \in s^{\prime} \tau$,

then $S$ has an E-unitary dense cover. Finally, if $S$ is orthodox and if $\tau$ satisfies the following condition

(**) for all $s \in S$ and $g \in s \tau$, there exists an inverse $s^{\prime}$ of $s$ such that $g^{-1} \in s^{\prime} \tau$,

then $S$ has an E-unitary orthodox cover.

Proof. Let $\tau=\alpha^{-1} \beta$ be the canonical factorization of $\tau$. Thus, we let $R$ be the graph of $\tau, R=\{(s, g) \in S \times G \mid g \in s \tau\}, \alpha: R \rightarrow S$ is defined by $(s, g) \alpha=s$ and $\beta: R \rightarrow G$ is defined by $(s, g) \beta=g$. We claim that $R$ is an E-unitary cover of $S$. The idempotents of $R$ are of the form $(e, 1)$ where $e \in E(S)$. Therefore $R$ is an E-semigroup and $\alpha$ is one-to-one on idempotents.

Let $(e, 1) \in E(R),(s, g) \in R$ and suppose that $(e, 1)(s, g) \in E(R)$. Then $g=1$ and, since $(s, g) \in R, s \in g \tau^{-1}=1 \tau^{-1}=E(S)$. Therefore $(s, g) \in E(R)$ and $R$ is E-unitary.

Suppose now that $S$ is E-dense and that $\tau$ satisfies $(*)$. Let $(s, g) \in R$. Then, by $(*)$, there exists $s^{\prime}$ such that $s s^{\prime} \in E(S)$ and $\left(s^{\prime}, g^{-1}\right) \in R$. It follows that $(s, g)\left(s^{\prime}, g^{-1}\right)=\left(s s^{\prime}, 1\right) \in E(R)$. Thus $R$ is E-dense by Proposition 1.1.

Finally, suppose that $S$ is regular and that $\tau$ satisfies condition $(* *)$. For each $(s, g) \in R$, by $(* *)$, there exists an inverse $s^{\prime}$ of $s$ such that $\left(s^{\prime}, g^{-1}\right) \in R$. Then $\left(s^{\prime}, g^{-1}\right)$ is an inverse of $(s, g)$, so $R$ is orthodox. 
Theorem 2.3 Let $S$ be an E-semigroup, and let $E=E(S)$. Then there exists a group $G$ and a relational morphism $\tau: E S E \rightarrow G$ such that $1 \tau^{-1}=E$. Furthermore, if $S$ is E-dense, then ESE is E-dense and $\tau$ satisfies $(*)$, and if $S$ is regular, then $E S E=S$ and $\tau$ satisfies $(* *)$.

Proof. Let $G$ be the free group with basis the set $S$. Let $\bar{S}$ be a copy of $S$ and let $s \mapsto \bar{s}$ be a bijection from $S$ onto $\bar{S}$. To avoid confusion with multiplication in $S$, we write elements of the free monoid $(S \cup \bar{S})^{*}$ as finite sequences; in particular, () will denote the empty sequence. It is well known that $G$ is the quotient of $(S \cup \bar{S})^{*}$ by the congruence generated by the relations $(s)(\bar{s})=()$ and $(\bar{s})(s)=()$. We denote by $\pi:(S \cup \bar{S})^{*} \rightarrow G$ the morphism induced by this congruence.

The bijection $s \mapsto \bar{s}$ can be extended into an involution of $(S \cup \bar{S})^{*}$ by setting $\overline{\bar{s}}=s$ for every $s \in S$ and

$$
\overline{\left(a_{1}, \ldots, a_{n}\right)}=\left(\bar{a}_{n}, \ldots, \bar{a}_{1}\right) \quad \text { for every } a_{1}, \ldots, a_{n} \in S \cup \bar{S} \text {. }
$$

Define a map $\sigma$ from $S \cup \bar{S}$ into the set of subsets of $S$ by setting

$$
\begin{aligned}
& s \sigma=E s E \\
& \bar{s} \sigma=\bigcup_{\left\{s^{\prime} \mid s s^{\prime} \in E \text { and } s^{\prime} s \in E\right\}}\left\{e \in E \mid e \leq_{\mathcal{L}} s^{\prime} s\right\} s^{\prime}\left\{e \in E \mid e \leq_{\mathcal{R}} s s^{\prime}\right\} .
\end{aligned}
$$

Then we have the following lemma.

Lemma 2.4 For every $s \in S$,

(a) $(s \sigma) E=E(s \sigma)=s \sigma$;

(b) $(\bar{s} \sigma) E=E(\bar{s} \sigma)=\bar{s} \sigma$;

(c) $(s \sigma)(\bar{s} \sigma) \subset E$ and $(\bar{s} \sigma)(s \sigma) \subset E$.

Proof. (a) is clear.

(b) Let $e \in E$ and $t \in \bar{s} \sigma$. Then $t=e_{1} s^{\prime} e_{2}$ for some $s^{\prime} \in S$ such that $s s^{\prime} \in E$ and $s^{\prime} s \in E$ and some $e_{1}, e_{2} \in E$ such that $e_{1} \leq_{\mathcal{L}} s^{\prime} s$ and $e_{2} \leq_{\mathcal{R}} s s^{\prime}$. It follows that $e e_{1} \leq_{\mathcal{L}} s^{\prime} s$ and $e_{2} e \leq_{\mathcal{R}} s s^{\prime}$. Thus

$$
e t=\left(e e_{1}\right) s^{\prime} e_{2} \in \bar{s} \sigma \quad \text { and } \quad t e=e_{1} s^{\prime}\left(e_{2} e\right) \in \bar{s} \sigma
$$

Therefore $E(\bar{s} \sigma) \subset \bar{s} \sigma$ and $(\bar{s} \sigma) E \subset \bar{s} \sigma$. Conversely, if $t=e_{1} s^{\prime} e_{2} \in \bar{s} \sigma$, then $e_{1} t=t=t e_{2}$, whence $t \in E(\bar{s} \sigma) \cap(\bar{s} \sigma) E$.

(c) By symmetry, it suffices to show that $(s \sigma)(\bar{s} \sigma) \subset E$. Let $t=e_{1} s e_{2} \in s \sigma$ (where $e_{1}, e_{2} \in E$ ) and let $t^{\prime}=e_{3} s^{\prime} e_{4} \in \bar{s} \sigma$ (where $s s^{\prime}, s^{\prime} s, e_{3}, e_{4} \in E, e_{3} \leq_{\mathcal{L}} s^{\prime} s$ and $\left.e_{4} \leq_{\mathcal{R}} s s^{\prime}\right)$. Then $s e_{2} e_{3} s^{\prime} \in E$ since

$$
\begin{aligned}
s e_{2} e_{3} s^{\prime} s e_{2} e_{3} s^{\prime} & =s e_{2} e_{3} e_{2} e_{3} s^{\prime} & & \left(\text { because } e_{3} \leq_{\mathcal{L}} s^{\prime} s\right) \\
& =s e_{2} e_{3} s^{\prime} & & \left(\text { because } e_{2} e_{3} \in E\right)
\end{aligned}
$$

It follows that $t t^{\prime}=e_{1}\left(s e_{2} e_{3} s^{\prime}\right) e_{4} \in E$.

Let $T$ be the set of all subsets of $S$ of the form $E X E$, where $X \subset S$. Then $T$ is a monoid with identity $E$ under multiplication of subsets. Lemma 2.4 shows 
that $\sigma$ is a map from $S \cup \bar{S}$ into $T$. Hence, it can be extended (in a unique way) into a monoid morphism $\sigma:(S \cup \bar{S})^{*} \rightarrow T$ by setting

$$
\begin{aligned}
() \sigma & =E \\
\left(a_{1}, \ldots, a_{n}\right) \sigma & =\left(a_{1} \sigma\right) \cdots\left(a_{n} \sigma\right) \quad \text { for every } a_{1}, \ldots, a_{n} \in S \cup \bar{S} .
\end{aligned}
$$

Lemma 2.5 For every $u \in(S \cup \bar{S})^{*}$ such that $u \pi=1, u \sigma \subset E$.

Proof. It is well known that $1 \pi^{-1}$ is the smallest submonoid $D$ of $(S \cup \bar{S})^{*}$ such that, for every $u_{1}, u_{2} \in(S \cup \bar{S})^{*}$ and for all $s \in S$,

$$
u_{1} u_{2} \in D \quad \text { implies } \quad u_{1}(s)(\bar{s}) u_{2} \in D \text { and } u_{1}(\bar{s})(s) u_{2} \in D .
$$

Let $D^{\prime}$ be the set of all $u \in D$ such that $u \sigma \subset E$. Since ()$\sigma=E$ and $(u v) \sigma=$ $(u \sigma)(v \sigma)$ for all $u, v \in(S \cup \bar{S})^{*}, D^{\prime}$ is a submonoid of $1 \pi^{-1}$. Furthermore, if $u_{1} u_{2} \in D^{\prime}$ and $s \in S$, then

$$
\begin{aligned}
\left(u_{1}(s)(\bar{s}) u_{2}\right) \sigma=\left(u_{1} \sigma\right)(s \sigma)(\bar{s} \sigma)\left(u_{2} \sigma\right) & \subset\left(u_{1} \sigma\right) E\left(u_{2} \sigma\right) \\
& \subset\left(u_{1} \sigma\right)\left(u_{2} \sigma\right)=\left(u_{1} u_{2}\right) \sigma \subset E,
\end{aligned}
$$

and symmetrically, $\left(u_{1}(\bar{s})(s) u_{2}\right) \sigma \subset E$. Therefore $u_{1}(s)(\bar{s}) u_{2}$ and $u_{1}(\bar{s})(s) u_{2}$ lie in $D^{\prime}$, whence $D^{\prime}=1 \pi^{-1}$.

Since for every $s \in S, s \sigma=E s E$, the relation $\sigma^{-1}: E S E \rightarrow(S \cup \bar{S})^{*}$ defined by $s \sigma^{-1}=\left\{u \in(S \cup \bar{S})^{*} \mid s \in u \sigma\right\}$ is a relational morphism. Let $\tau=\sigma^{-1} \pi$. Then $\tau: E S E \rightarrow G$ is a relational morphism. The following diagram may help the reader to visualize the mappings and relations we are considering.

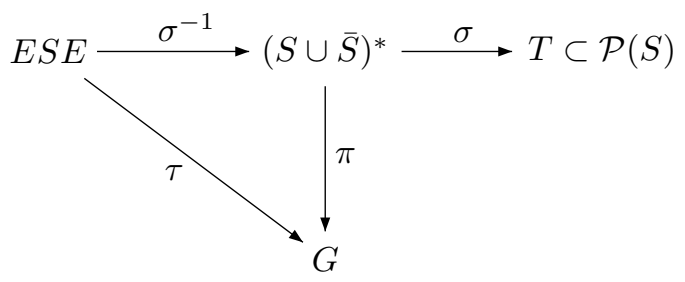

We claim that $1 \tau^{-1}=E$. First, if $e \in E$, then ()$\in e \sigma^{-1}$ and ()$\pi=1$. Therefore $1 \in e \tau$, and thus $E \subset 1 \tau^{-1}$. Conversely, let $s \in 1 \tau^{-1}$. Then there exists $u \in(S \cup \bar{S})^{*}$ such that $u \pi=1$ and $s \in u \sigma$. By Lemma 2.5, $u \sigma \subset E$ and thus $s \in E$. This completes the proof of the first part of Theorem 2.3.

It remains to show that if $S$ is E-dense (resp. regular), then $\tau$ satisfies $(*)$ (resp. (**)). Let us first assume that $S$ is E-dense. Let $s \in E S E$ and let $g \in s \tau$. Let $u \in(S \cup \bar{S})^{*}$ be such that $s \in u \sigma$ and $u \pi=g$. Then, by Lemma 2.5, $(u \sigma)(\bar{u} \sigma) \subset E$ and $(u \bar{u}) \pi=1$. Since $S$ is E-dense, $\bar{u} \sigma$ is non-empty: indeed, for every $t \in S$, by Proposition 1.1, there exists an element $t^{\prime}$ such that $t t^{\prime} \in E$ and $t^{\prime} t \in E$, and thus $\bar{t} \sigma \neq \emptyset$. Let $s^{\prime} \in \bar{u} \sigma$. Then $s s^{\prime} \in(u \sigma)(\bar{u} \sigma) \subset E$ and thus $s s^{\prime} \in E$. On the other hand, $g^{-1} \in s^{\prime} \tau$.

Now let us assume that $S$ is regular, that is, $S$ is orthodox. For each $s \in S$, let $V(s)$ be the set of inverses of $s$. It is well-known that in orthodox semigroups $V(s) V(t) \subset V(t s)$ [20]. Again we may consider $u \in(S \cup \bar{S})^{*}$ such that $s \in u \sigma$ and $u \pi=g$, say $u=\left(a_{1}, \ldots, a_{n}\right)$ with $a_{1}, \ldots, a_{n} \in S \cup \bar{S}$. So $s=s_{1} \ldots s_{n}$ for 
some $s_{i} \in\left(a_{i}\right) \sigma(1 \leq i \leq n)$. Since $V\left(s_{n}\right) \ldots V\left(s_{1}\right) \subset V(s)$, it suffices to show that, for each $t \in S$, each element of $(t) \sigma$ (resp. $(\bar{t}) \sigma)$ has an inverse in $(\bar{t}) \sigma$ (resp. $(t) \sigma)$. Indeed this will imply that $\bar{u} \sigma$ contains an inverse $s^{\prime}$ of $s$. But $\bar{u} \pi=g^{-1}$, so $\tau$ satisfies $(* *)$.

So let $t \in S$ and let $x \in(t) \sigma$. Then $x=$ etf for some $e, f \in E$. Let $t^{\prime} \in V(t)$. Then $x^{\prime}=f t^{\prime} e \in V(x)$ and $x^{\prime} \in(\bar{t}) \sigma$ since $x^{\prime}=\left(f t^{\prime} t\right) t^{\prime}\left(t t^{\prime} e\right)$. Now let $y \in(\bar{t}) \sigma$. Then $y=\left(e t^{\prime} t\right) t^{\prime}\left(t t^{\prime} f\right)$ for some $e, f$ and $t^{\prime}$ such that $e$, $f, t t^{\prime}$ and $t^{\prime} t$ are idempotents. Let $t^{\prime \prime} \in V\left(t^{\prime}\right)$ and $y^{\prime}=\left(f t t^{\prime}\right) t^{\prime \prime}\left(e t^{\prime} t\right)$. Since $y^{\prime} \in V\left(t t^{\prime} f\right) V\left(t^{\prime}\right) V\left(e t^{\prime} t\right), y^{\prime}$ is an inverse of $y$, and since $y^{\prime}=(f) t\left(t^{\prime} t^{\prime \prime} e t^{\prime} t\right)$, $y^{\prime} \in(t) \sigma$.

We can now conclude the proof of Theorem 2.1. Let $S$ be an E-semigroup. Then $M=S^{1}$ is an E-monoid. By Proposition 2.2 and Theorem 2.3, $E M E=M$ has an E-unitary cover $\varphi: \hat{M} \rightarrow M$. Since $\varphi$ is one-to-one on idempotents the restriction of $\varphi$ to $\hat{S}=S \varphi^{-1}$ is also a covering $\varphi: \hat{S} \rightarrow S$, and $\hat{S}$ is E-unitary. Furthermore, if $S$ is E-dense (resp. regular), then so are $M, \hat{M}$ and $\hat{S}$.

Since a covering is one-to-one on idempotents, Fountain's result follows immediately [5] (see McAlister [11, 12] and O'Caroll [15], or [9] for the inverse case).

\section{Corollary 2.6}

(1) Every E-commutative semigroup has an E-commutative unitary cover.

(2) Every E-commutative dense semigroup has an E-commutative unitary dense cover.

(3) Every inverse semigroup has an E-unitary inverse cover.

We conclude this section by pointing out an interesting property of the above construction of an E-unitary cover. Let $\alpha_{S}: \hat{S} \rightarrow S$ be the covering constructed in the proof of Theorem 2.1. It has the following property.

Proposition 2.7 For every morphism $\varphi: S \rightarrow T$, there exists a morphism $\hat{\varphi}: \hat{S} \rightarrow \hat{T}$ such that $\hat{\varphi} \alpha_{T}=\alpha_{S} \varphi$. However, $\hat{\varphi}$ needs not be onto even if $\varphi$ is.

Proof. Let $\pi_{S}:(S \cup \bar{S})^{*} \rightarrow G_{S}$ and $\pi_{T}:(T \cup \bar{T})^{*} \rightarrow G_{T}$ be the canonical projections onto the free groups with bases, respectively, the sets $S$ and $T$. It is straightforward that the morphism $\varphi: S \rightarrow T$ can be extended canonically to morphisms $\varphi: \mathcal{P}(S) \rightarrow \mathcal{P}(T)$ and $\varphi:(S \cup \bar{S})^{*} \rightarrow(T \cup \bar{T})^{*}$, and that it induces a morphism $\varphi_{1}: G_{S} \rightarrow G_{T}$ such that $\pi_{S} \varphi_{1}=\varphi \pi_{T}$.

Let now $\sigma_{S}\left(\right.$ resp. $\sigma_{T}$ ) be the map from $S$ (resp. $T$ ) into the set of subsets of $(S \cup \bar{S})^{*}\left(\right.$ resp. $\left.(T \cup \bar{T})^{*}\right)$ defined in the proof of Theorem 2.3. For each $s \in S$, we have $(s) \sigma_{S} \varphi \subset(s \varphi) \sigma_{T}$ and $(\bar{s}) \sigma_{S} \varphi \subset(\overline{s \varphi}) \sigma_{T}$. Note that here equality does not necessarily hold since, in particular, $E(S) \varphi$ may be strictly contained in $E(T)$. Then, for each $u \in(S \cup \bar{S})^{*}, u \sigma_{S} \varphi \subset u \varphi \sigma_{T}$. If $\tau_{S}=\sigma_{S}^{-1} \pi_{S}$ and $\tau_{T}=\sigma_{T}^{-1} \pi_{T}$, we have, for every $s$ in $S$ :

$$
s \tau_{S} \varphi_{1}=s \sigma_{S}^{-1} \pi_{S} \varphi_{1}=s \sigma_{S}^{-1} \varphi \pi_{T} \subset s \varphi \sigma_{T}^{-1} \pi_{T}=s \varphi \tau_{T} .
$$


But $\hat{S}$ and $\hat{T}$ are respectively the graphs of $\tau_{S}$ and $\tau_{T}$, and $\alpha_{S}$ and $\alpha_{T}$ are the projections of these graphs onto their first components. So, letting $(s, g) \hat{\varphi}=\left(s \varphi, g \varphi_{1}\right)$ for each $(s, g) \in \hat{S}$ defines a morphism $\hat{\varphi}: \hat{S} \rightarrow \hat{T}$ that verifies $\hat{\varphi} \alpha_{T}=\alpha_{S} \varphi$.

\section{The structure of E-unitary dense semigroups}

According to [9], we define the fundamental group $\pi_{1}(S)$ of a semigroup $S$ as the quotient of the free group $F(S)$ with basis $S$ by the relations $(s)(t)=$ $(s t)$, for every $s, t \in S$. Let $\iota: S \rightarrow F(S)$ be the natural embedding and let $\pi: F(S) \rightarrow \pi_{1}(S)$ be the natural surjective group morphism. Then the map $\eta=\imath \pi: S \rightarrow \pi_{1}(S)$ is a morphism which is characterized by the following universal property. For every semigroup morphism $\gamma: S \rightarrow G$ into a group $G$, there exists a unique group morphism $\varphi: \pi_{1}(S) \rightarrow G$ such that $\gamma=\eta \varphi$. Moreover, if $S \gamma$ generates $G$ as a group, then $\varphi$ is surjective. It follows that, when $S$ is E-dense, $\pi_{1}(S)$ is the maximal quotient group of $S$ [9].

We can now state and prove a theorem which generalizes an analogous result for E-commutative semigroups (Margolis and Pin [9]).

Theorem 3.1 Let $S$ be a non-empty E-semigroup. The following conditions are equivalent:

(1) There exist a group $G$ and a surjective morphism $\varphi: S \rightarrow G$ such that $1 \varphi^{-1}=E(S)$.

(2) There exists a surjective morphism $\psi: S \rightarrow \pi_{1}(S)$ such that $1 \psi^{-1}=E(S)$.

(3) The morphism $\eta: S \rightarrow \pi_{1}(S)$ is surjective and satisfies $1 \eta^{-1}=E(S)$.

(4) $S$ is E-unitary dense.

Proof. Let $E=E(S)$. Clearly (3) implies (2) and (2) implies (1). We prove that (1) implies (4). If $e, e s \in E$ for some $e, s \in S$, then $1=(e s) \varphi=(e \varphi)(s \varphi)=$ $s \varphi$, whence $s \in 1 \varphi^{-1}=E$. Therefore $S$ is E-unitary. Moreover, for every $s \in S$, there exists $t \in S$ such that $t \varphi=(s \varphi)^{-1}$. Therefore $(s t) \varphi=1$, whence $s t \in E$. Thus $S$ is E-dense.

It remains to show that (4) implies (3). Let $S$ be an E-unitary dense semigroup. For every $s \in S$, let $X(s)$ be the smallest subset $X$ of $S^{1}$ containing $s$ and such that

(a) for all $s_{1}, s_{2} \in S^{1}$ and $e \in E, s_{1} s_{2} \in X$ implies $s_{1} e s_{2} \in X$,

(b) for all $s_{1}, s_{2} \in S^{1}$ and $e \in E, s_{1} e s_{2} \in X$ implies $s_{1} s_{2} \in X$.

We define an equivalence relation $\sim$ on $S$ by letting

$$
s \sim t \quad \text { if and only if } \quad X(s)=X(t) .
$$

It is easy to verify that $s \sim t$ if and only if there exist three sequences of elements of $S,\left(u_{0}, \ldots, u_{2 k+1}\right),\left(u_{0}^{\prime}, \ldots, u_{2 k+1}^{\prime}\right)$ and $\left(u_{0}^{\prime \prime}, \ldots, u_{2 k+1}^{\prime \prime}\right)$, and a sequence of elements of $E,\left(e_{1}, \ldots, e_{2 k+1}\right)$, such that

$$
\begin{aligned}
& u_{0}=s, \quad u_{2 k+1}=t \quad \text { and } \\
& u_{2 i}=u_{2 i}^{\prime} u_{2 i}^{\prime \prime}, \quad u_{2 i+1}=u_{2 i}^{\prime} e_{2 i} u_{2 i}^{\prime \prime} \quad(0 \leq i \leq k) \\
& u_{2 i+1}=u_{2 i+1}^{\prime} e_{2 i+1} u_{2 i+1}^{\prime \prime}, \quad u_{2 i+2}=u_{2 i+1}^{\prime} u_{2 i+1}^{\prime \prime} \quad(0 \leq i \leq k-1)
\end{aligned}
$$


It follows that $\sim$ is a congruence.

We claim that $X(e)=E$ for every $e \in E$. Indeed $1 \cdot e \cdot 1=e \in X(e)$ and thus, by (b), $1 \in X(e)$. It follows by (a) that $E \subset X(e)$. Conversely, $e \in E$ and, by Proposition 1.2, $E$ satisfies (a) and (b). Thus, $X(e) \subset E$. In particular, all idempotents of $S$ are congruent modulo $\sim$. Conversely, if $s \sim e$ for some $e \in E$, then $s \in X(e)=E$ and $s$ is idempotent.

Let $\pi: S \rightarrow S / \sim$ be the natural projection. We claim that $S / \sim=\pi_{1}(S)$ and $\pi=\eta$. First, since $S$ is non-empty and E-dense, it contains at least one idempotent $e$. Since $e s \sim s \sim s e, e \pi=1$ is an identity for $S / \sim$. Furthermore, since $S$ is E-dense, there exists for each $s \in S$ an $s^{\prime} \in S$ such that $s s^{\prime} \in E$ and $s^{\prime} s \in E$. Therefore $\left(s s^{\prime}\right) \pi=\left(s^{\prime} s\right) \pi=1$. Thus $S / \sim$ is a group. By the preceding paragraph, we have

$$
1 \pi^{-1}=\{s \in S \mid s \sim e\}=E .
$$

Finally, let $\gamma: S \rightarrow G$ be a surjective semigroup morphism onto a group $G$. The constructive definition of $\sim$ shows that, if $s \sim t$, then $s \gamma=t \gamma$ (since for every $e \in E, e \gamma=1$ ). Therefore there exists a unique surjective semigroup morphism $\varphi: S / \sim \rightarrow G$ such that $\gamma=\pi \varphi$. In fact $\varphi$ is a group morphism and $\pi$ satisfies the same universal property as $\eta$. So $S / \sim=\pi_{1}(S)$ and $\pi=\eta$.

An important tool introduced in [9] and later extended in [28] is the use of categories as a generalization of monoids. In the case of semigroups, the natural extension is the notion of "category without identity", also called "semigroupoid" by Tilson. Since both terms seem to be unfortunate - the first one is too negative and the second one too technical-, we take the risk of introducing the new terminology of quiver for these objects. "Quiver" is the translation of the French word carquois, which was originally chosen by the authors. It is meant to be as descriptive as possible, in the sense that a quiver "contains a bunch of arrows". ${ }^{2}$

Formally, a quiver $C$ is given by

(a) a $\operatorname{set}^{3} \mathrm{Ob}(C)$ of objects,

(b) for each pair $(u, v)$ of objects, a set $C(u, v)$ of arrows,

(c) for each triple $(u, v, w)$ of objects, a mapping from $C(u, v) \times C(v, w)$ into $C(u, w)$ which associates to each $p \in C(u, v)$ and $q \in C(v, w)$ the composition $p+q \in C(u, w)$.

The additive notation is convenient because it will allow us to write group actions multiplicatively, but it does not imply commutativity. Composition is assumed to be associative (when defined).

Note that a semigroup is simply a quiver with exactly one object. A quiver $C$ is said to be locally idempotent (resp. locally commutative, etc.) if, for each object $u$ of $C$, the semigroup $C(u, u)$ is idempotent (resp. commutative, etc.). It is regular if, for each arrow $p \in C(u, v)$, there exists an arrow $q \in C(v, u)$ such that $p+q+p=p$, and it is connected if $C(u, v) \neq \emptyset$ for each pair $(u, v)$ of objects of $C$.

\footnotetext{
${ }^{2}$ As is attested by the classical literature: "I perceived it to be a human creature not six inches high, with a bow and arrows in his hands, and a quiver at his back." [21]

${ }^{3}$ Since we consider quivers and categories as algebraic objects, there is no need to introduce the usual distinction between sets and classes. In the terminology of category theory, we consider only "small" categories.
} 
In the usual terminology of category theory, what we call here arrows is called morphisms. Since we want to view quivers as generalized semigroups, we prefer to reserve the term morphism for what is usually called functors. More precisely, a morphism $\varphi: C \rightarrow D$ between two quivers $C$ and $D$ is given by:

(1) a function $\varphi: O b(C) \rightarrow O b(D)$ and

(2) for every $u, v \in O b(C)$, a function $\varphi_{u, v}: C(u, v) \rightarrow D(u \varphi, v \varphi)$ such that, for every $u, v, w \in O b(C)$ and for every $p \in C(u, v)$ and $q \in C(v, w)$,

$$
p \varphi_{u, v}+q \varphi_{v, w}=(p+q) \varphi_{u, w} .
$$

Subscripts are usually omitted, and the last formula is written as

$$
p \varphi+q \varphi=(p+q) \varphi \text {. }
$$

An automorphism $\varphi$ of a quiver $C$ is defined as usual. In particular, $\varphi$ is then a permutation of $O b(C)$ and is a bijection between $C(u, v)$ and $C(u \varphi, v \varphi)$. We denote by $A u t(C)$ the group of automorphisms of $C$. An action of a group $G$ on $C$ is given by a group morphism from $G$ into $A u t(C)$. In this case we write $g u$ (resp. $g p$ ) the result of the action of $g$ on the object $u$ (resp. the arrow $p$ ). Note the following identities.

(1) $g(p+q)=g p+g q$ for all $g \in G, p \in C(u, v)$ and $q \in C(v, w)$.

(2) $(g h) p=g(h p)$ for all $g, h \in G$ and $p \in C(u, v)$.

Whenever a group $G$ acts on a quiver $C$, a quotient quiver $C / G$ is defined, with object set $O b(C) / G$, that is, the set of disjoint subsets of $O b(C)$ of the form $G u(u \in O b(C))$, and with arrow sets

$$
C / G(G u, G v)=\left\{G p \mid p \in C\left(u^{\prime}, v^{\prime}\right), u^{\prime} \in G u, v^{\prime} \in G v\right\} .
$$

Composition of consecutive arrows $G p$ and $G q$ (that is, $p \in C(u, v)$ and $q \in$ $C(g v, w)$ for some objects $u, v$ and $w$ and some element $g$ of $G$ ) is given by, $G p+G q=G\left(p+g^{-1} q\right)$.

The following results are proved in [9] for categories, but their proofs can be readily adapted to the case of quivers.

If a group $G$ acts transitively without fixpoints on a quiver $C$, then the quiver $C / G$ is a semigroup. Let $u$ be any object of $C$ and let

$$
C_{u}=\{(p, g) \mid g \in G, p \in C(u, g u)\} .
$$

Then $C_{u}$ is a semigroup for the multiplication defined by $(p, g)(q, h)=(p+$ $g q, g h)$, and we can state

Proposition 3.2 Let $G$ be a group acting transitively without fixpoints on a quiver $C$. Then, for all $u \in O b(C)$, the semigroup $C_{u}$ is isomorphic to $C / G$.

Let $\varphi: S \rightarrow T$ be a semigroup morphism. Define a quiver $C$ with $O b(C)=T$ and, for $u, v \in T, C(u, v)=\{(u, s, v) \in T \times S \times T \mid u(s \varphi)=v\}$. Composition is given by $(u, s, v)+(v, t, w)=(u, s t, w)$. Clearly it is associative. So $C$ is a quiver which we call the derived quiver of $\varphi$. (Tilson's definition of the derived quiver of a semigroup morphism [28] is the quotient of $C$ by a certain quiver congruence. But for our purposes this simplified definition will be sufficient.)

Now the derived covering of $\varphi$ is the morphism $\Phi: C \rightarrow S$ defined by the following conditions: 
(a) for all $u \in O b(C), u \Phi$ is the only object of $S$,

(b) for all $(u, s, v) \in C(u, v),(u, s, v) \Phi=s$.

Proposition 3.3 [9] Let $\varphi: S \rightarrow G$ be a morphism into a group and let $\Phi: C \rightarrow$ $S$ be the derived covering of $\varphi$. Then $G$ acts transitively without fixpoints on $C$ and $S$ is isomorphic (as a semigroup) to $C / G$.

Theorem 3.4 Let $S$ be an E-semigroup. The following conditions are equivalent.

(1) $S$ is E-unitary dense.

(2) $S$ is isomorphic to $C / G$ where $G$ is a group acting transitively without fixpoints on some connected locally idempotent quiver $C$.

Proof. The proof essentially mimics the proofs of Propositions 3.12 and 3.14 of [9].

(1) $\Longrightarrow(2)$. By Theorem 3.1, there exists a morphism $\varphi: S \rightarrow G$ onto a group such that $1 \varphi^{-1}=E(S)$. By Proposition $3.3, S$ is isomorphic to $C / G$ where $C$ is the derived quiver of $\varphi$. Since $\varphi$ is onto, then, for every $g, h \in G$, there exists $s \in S$ such that $s \in\left(g^{-1} h\right) \varphi^{-1}$. It follows that $(g, s, h) \in C(g, h)$ and $C$ is connected. Next, for every $g \in G$,

$$
C(g, g)=\{(g, s, g) \mid g(s \varphi)=g\}
$$

is a semigroup isomorphic to $1 \varphi^{-1}=E(S)$. Thus $C$ is locally idempotent.

$(2) \Longrightarrow(1)$. By Proposition $3.2, C / G$ is isomorphic to $C_{1}$. Now $(p, g)$ is an idempotent in $C_{1}$ if and only if $p=p+g p$ and $g=g^{2}$, that is, if and only if $g=1$ and $p \in C(1,1)$ is idempotent. Since $C$ is locally idempotent, it follows that $E\left(C_{1}\right)=C(1,1) \times\{1\}$ and $C_{1}$ is an E-semigroup. Assume now that $(p, g)(q, 1)=(p+g q, g) \in E\left(C_{1}\right)$. Then $g=1$ and $p \in C(1,1)$. Thus, $C_{1}$ is E-unitary.

Finally let $(p, g) \in C_{1}$. Since $C$ is connected, there exists a morphism $q$ in $C\left(1, g^{-1}\right)$. Then $(p, g)\left(q, g^{-1}\right)=(p+g q, 1) \in E\left(C_{1}\right)$. Thus $C_{1}$ is E-dense.

Remark. Theorem 3.4 above was already formulated in the regular (orthodox) case by Szendrei [25].

\section{The finite case}

The class of finite E-semigroups was described in a paper by Jones and Szendrei [6]. This section is only a rewriting of her work, and it is included here in order to provide a complete presentation.

Let $\mathbf{E S}$ be the class of all finite E-semigroups. ES is the variety of finite semigroups which is defined by the pseudo-identity $\left(x^{\omega} y^{\omega}\right)^{\omega}=x^{\omega} y^{\omega}$ (see Eilenberg [4], or Pin [17]). For each variety of finite bands $\mathbf{V}$, we let $\mathbf{E S}(\mathbf{V})$ be the variety of finite E-semigroups whose band of idempotents is in $\mathbf{V}$.

Note that in a finite E-semigroup, the set of idempotents is always dense, since every element can be completed on the right and on the left into an idempotent of the minimal ideal. So the structure of the E-unitary elements of ES is described in Section 3 above. 
The covering theorem (Theorem 2.1 above) can be applied to finite E-semigroups, but the E-unitary cover that is constructed in its proof is infinite. However, the theorem can be relativized to finite semigroups, thanks to the following result of Birget, Margolis and Rhodes [3]. Note that in the case of E-commutative monoids, the result is a consequence of Margolis and Pin [10] and Ash [1].

Theorem 4.1 Let $S \in$ ES. There exist a finite group $G$ and a relational morphism $\tau: S \rightarrow G$ such that $1 \tau^{-1}=E(S)$.

Proof. The exact result proved in [3] is that, if $S \in \mathbf{E S}$, then $K(S)=E(S)$, where $K(S)$ (the "kernel" of $S$, or the subsemigroup of "type II" elements of $S$ ) is the intersection of the $1 \sigma^{-1}$ for all relational morphisms $\sigma$ from $S$ into a finite group. This implies the announced result. Indeed (see [18]), since there are only finitely many subsemigroups of $S$, one can select finitely many such relational morphisms, $\sigma_{1}: S \rightarrow G_{1}, \ldots, \sigma_{k}: S \rightarrow G_{k}$, in such a way that, for any relational morphism $\sigma$ from $S$ into a finite group $G, 1 \sigma^{-1}=1 \sigma_{i}^{-1}$ for some $1 \leq i \leq k$. The direct product $\tau=\left(\sigma_{1}, \ldots, \sigma_{k}\right): S \rightarrow G_{1} \times \cdots \times G_{k}$ is then a relational morphism and $1 \tau^{-1}=\bigcap_{i=1}^{k} 1 \sigma_{i}^{-1}=K(S)=E(S)$.

Corollary 4.2 Let $S \in \mathbf{E S}(\mathbf{V})$.

(1) $S$ has a finite E-unitary cover $R$ in $\mathbf{E S}(\mathbf{V})$.

(2) If $S$ is regular (that is, $S$ is orthodox), then $R$ can be chosen to be regular too.

Proof. Let $\tau: S \rightarrow G$ be a relational morphism into a finite group $G$ such that $1 \tau^{-1}=E(S)$. If $R$ is the graph of $\tau$, then $R$ is finite and the same proof as for Proposition 2.2 shows that $R$ is an E-unitary cover of $S$. Suppose next that $S$ is regular, and let $(s, g) \in R$. Let $s^{\prime}$ be an inverse of $s$ and let $g^{\prime} \in s^{\prime} \tau$. There exists an integer $k \geq 1$ such that $\left(g g^{\prime}\right)^{k}=1$. Then

$$
g^{-1}=g^{\prime}\left(g g^{\prime}\right)^{k-1} \in s^{\prime} \tau\left(s \tau s^{\prime} \tau\right)^{k-1} \subset\left(s^{\prime}\left(s s^{\prime}\right)^{k-1}\right) \tau=s^{\prime} \tau .
$$

So $\left(s^{\prime}, g^{-1}\right) \in R$ and hence $\tau$ satisfies condition (**) of Proposition 2.2.

Theorem 4.1 above also implies the following characterization of the varieties $\mathbf{E S}(\mathbf{V})$, where $\mathbf{V}$ is a variety of finite idempotent semigroups, and $\mathbf{V}^{-1} \mathbf{G}$ is the class of all semigroups $S$ for which there exists a relational morphism $\tau$ from $S$ into a finite group such that $1 \tau^{-1} \in \mathbf{V}$.

Corollary 4.3 Let $S$ be a finite semigroup and let $\mathbf{V}$ be a variety of finite bands. The following are equivalent.

(1) $S \in \mathbf{E S}(\mathbf{V})$.

(2) $K(S)=E(S)$ and $K(S) \in \mathbf{V}$.

(3) $S \in \mathbf{V}^{-1} \mathbf{G}$.

(4) $S \in \mathbf{V} * \mathbf{G}$.

(5) $S$ divides some finite orthodox E-unitary semigroup in $\mathbf{E S}(\mathbf{V})$. 
(6) $S$ divides some finite orthodox semigroup in $\mathbf{E S}(\mathbf{V})$.

Proof. (1) implies (2) and (2) implies (3), as was proved in Theorem 4.1 above. (4) implies (5), as one can readily verify that the semidirect product of an idempotent semigroup by a group is E-unitary and orthodox. (5) implies (6) and (6) implies (1), trivially.

The fact that (3) implies (4) is a consequence of a result of Szendrei [25] (which itself was a generalization of a theorem of Weiss and Thérien [29]), according to which the finite quivers that are locally in $\mathbf{V}$ divide a semigroup in $\mathbf{V}$, and of Tilson's derived category theorem [28]. Let $\tau: S \rightarrow G$ be a relational morphism into a group $G$, such that $1 \tau^{-1} \in \mathbf{V}$. Let $R_{\tau}$ be the graph of $\tau$, and let $\tau=\alpha^{-1} \beta$ be its canonical factorization (see section 1). It is easy to verify that $1 \beta^{-1}$ is isomorphic to $1 \tau^{-1}$ and hence lies in $\mathbf{V}$. So the derived quiver of $\beta$ defined section 3 is locally in $\mathbf{V}$. Jones and Szendrei's result and Tilson's theorem then show that $R_{\tau}$ lies in $\mathbf{V} * \mathbf{G}$, and hence, so does $S$.

\section{Acknowledgements}

The authors wish to thank M. Szendrei and H. Mitsch for useful comments on an earlier version of this paper.

\section{References}

[1] C. Ash. Finite semigroups with commuting idempotents, J. Austral. Math. Soc. (Series A) 43 (1987).

[2] J. Berstel et D. Perrin. Theory of codes, Academic Press (London), 1985.

[3] J.-C. Birget, S. Margolis and J. Rhodes. Finite semigroups whose idempotents commute or form a subsemigroup. Bull. Austral. Math. Soc. 41 (1990), 161-184.

[4] S. Eilenberg. Automata, Languages and Machines, vol. B, Academic Press (New-York), 1976.

[5] J. Fountain. E-unitary dense covers of E-dense monoids. Bull. London Math. Soc. 22 (1990), 353-358.

[6] P. R. Jones and M. B. Szendrei. Local varieties of completely regular monoids, J. Algebra 150 (1992), 1-27.

[7] J. Kadourek and M. Szendrei. A new approach in the theory of orthodox semigroups, Semigroup Forum 40 (1990), 257-296.

[8] G. Lallement. Semigroups and Combinatorial Applications, Wiley (NewYork), 1979.

[9] S. Margolis and J.-E. Pin. Inverse semigroups and extensions of groups by semilattices, J. Algebra 110 (1987), 277-297. 
[10] S. Margolis and J.-E. Pin. Inverse semigroups and varieties of finite semigroups, J. Algebra 110 (1987), 306-323.

[11] D. McAlister. Groups, semilattices and inverse semigroups, Trans. Amer. Math. Soc. 192 (1974), 227-244.

[12] D. McAlister. Groups, semilattices and inverse semigroups, II, Trans. Amer. Math. Soc. 196 (1974), 251-270.

[13] D. McAlister and N. Reilly. E-unitary covers for inverse semigroups, Pacific J. Math. 68 (1977), 161-174.

[14] H. Mitsch. Subdirect products of E-inversive semigroups, J. Austral. Math. Soc. 48 (1990), 68-78.

[15] L. O'Caroll. Embedding theorems for proper inverse semigroups, J. Algebra 42 (1976), 26-40.

[16] M. Petrich. Inverse semigroups, Wiley (New York), 1984.

[17] J.-E. Pin. Variétés de langages formels, Masson (Paris), 1984, and Varieties of formal languages, North Oxford Academic (London), 1986 and Plenum (New York), 1986.

[18] J.-E. Pin. On a conjecture of Rhodes, Semigroup Forum 39 (1989), 1-15.

[19] J.-E. Pin. Relational morphisms, transductions and operations on languages, in J.-E. Pin (ed.), Formal properties of finite automata and applications, Lect. Notes in Comp. Sci. 386, Springer, 1989, 120-137.

[20] N. Reilly and H. Scheiblich. Congruences on regular semigroups, Pacific Journ. Math. 23 (1967), 349-360.

[21] (L. Gulliver). Travels into several Remote Nations of the World, B. Motte (London), 1726.

[22] M. Szendrei. On a pull-back diagram for orthodox semigroups, Semigroup Forum 20 (1980), 1-10. Correction 25 (1982), 311-324.

[23] M. Szendrei. E-unitary R-unipotent semigroups, Semigroup Forum 32 (1985), 87-96.

[24] M. Szendrei. E-unitary regular semigroups, Proc. Royal Soc. Edinburgh 106A (1987), 89-102.

[25] M. Szendrei. A generalization of McAlister's P-theorem for E-unitary regular semigroups. Acta Sci. Math. (Szeged) 51 (1987), 229-249.

[26] K. Takizawa. E-unitary R-unipotent semigroups, Bull. Tokyo Gakugei Univ., Series IV, 30 (1978), 21-33.

[27] K. Takizawa. Orthodox semigroups and E-unitary regular semigroups, Bull. Tokyo Gakugei Univ., Series IV, 31 (1979), 41-43.

[28] B. Tilson. Categories as algebras : an essential ingredient in the theory of monoids, J. Pure and Applied Algebra 48 (1987), 3-198. 
[29] A. Weiss and D. Thérien. Varieties of finite categories, RAIRO Inform. Théor. Appl. 20 (1986), 357-366. 